\title{
LA-UR-13-21653
}

Approved for public release; distribution is unlimited.

Title:

Author(s):

Intended for:

\section{Beam dynamics modeling in RFQ with CST Particle Studio}

Kurennoy, Sergey S.

Distribution to external collaborators

\section{- LOSAlamos \\ EST. 1943}

Disclaimer:

Los Alamos National Laboratory, an affirmative action/equal opportunity employer,is operated by the Los Alamos National

Security, LLC for the National NuclearSecurity Administration of the U.S. Department of Energy under contract DE-AC52-06NA25396. By approving this article, the publisher recognizes that the U.S. Government retains nonexclusive, royalty-free license to publish or reproduce the published form of this contribution, or to allow others to do so, for U.S. Government purposes.

Los Alamos National Laboratory requests that the publisher identify this article as work performed under the auspices of the

U.S. Departmentof Energy. Los Alamos National Laboratory strongly supports academic freedom and a researcher's right to publish; as an institution, however, the Laboratory does not endorse the viewpoint of a publication or guarantee its technical correctness. 
Los Alamos

NATIONAL LABORATORY

\section{Technical Note}

Accelerator Operation and Technology

AOT-HPE: High-Power Electrodynamics
To/MS: Distribution

From/MS: Sergey Kurennoy, AOT-HPE / H817

Phone/Fax: 505-665-1459 / 505-665-2904

E-mail: kurennoy@lanl.gov

Symbol: AOT-HPE: 13-005 (TN)

Date: February 27, 2013

\section{SUBJECT: Beam dynamics modeling in RFQ with CST Particle Studio.}

Starting with a detailed engineering CAD model, an electromagnetic analysis of the 4-rod FNAL RFQ was performed [1] using the CST MicroWave Studio (MWS) [2]. The MWS-calculated RF fields are used for PIC modeling of beam dynamics in the RFQ with CST Particle Studio (PS) [2]. This technical note describes our PS modeling and summarizes its results for the FNAL RFQ.

Distribution:

AOT-HPE File AOT-HPE

B.E. Carlsten

J.L. Erickson

S. Nath

I. Draganic

M.J. Borden

J.F. O'Hara

E.R. Olivas

Y.K. Batygin

R.C. McCrady

D.E. Rees

J.T. Lyles

\begin{tabular}{|c|c|c|}
\hline AOT-DO & MS H817 & $\begin{array}{l}\text { (e-mail copy) } \\
\text { (e-mail copy) }\end{array}$ \\
\hline AOT-DO & MS H809 & (e-mail copy) \\
\hline AOT-DO & MS H809 & (e-mail copy) \\
\hline AOT-IC & MS H817 & (e-mail copy) \\
\hline AOT-MDE & MS H838 & (e-mail copy) \\
\hline AOT-MDE & MS H838 & (e-mail copy) \\
\hline AOT-MDE & MS H838 & (e-mail copy) \\
\hline AOT-OPS & MS H817 & (e-mail copy) \\
\hline AOT-OPS & MS H817 & (e-mail copy) \\
\hline AOT-RFE & MS H827 & (e-mail copy) \\
\hline AOT-RFE & MS H827 & (e-mail copy) \\
\hline
\end{tabular}

(e-mail copy)

(e-mail copy)

(e-mail copy)

(e-mail copy)

e-mail copy)

e-mail copy)

(e-mail copy)

(e-mail copy)

(e-mail copy)

(e-mail copy) 


\section{Introduction.}

A new RFQ-based front end is planned for the LANSCE accelerator complex, initially to replace the existing Cockroft-Walton proton injection [3]. To be fully prepared to evaluate its design, we studied in detail a 4-rod RFQ, which was recently designed for Fermilab at the Goethe University (Frankfurt, Germany), fabricated by Kress $\mathrm{GmbH}$, and then installed and operated at FNAL [4]. Many parameters of the FNAL RFQ are similar to those for LANSCE: the RF frequency is $201.25 \mathrm{MHz}$, the input and output energies are 35 and $750 \mathrm{keV}$, respectively. The major difference is the duty factor, which is very low, $0.12 \%$, for the Fermilab design, but should be up to $15 \%$ for LANL.

The electromagnetic (EM) analysis of the FNAL RFQ was performed in [1] with the CST MicroWave Studio (MWS) [2] using the original RFQ CAD files provided by Kress GmbH. The calculated RF fields are used to simulate the beam propagation through the RFQ using a particle-in-cell (PIC) solver in the CST Particle Studio (PS) [2]. The beam dynamics modeling with PS is described in this technical note.

\section{Beam dynamics simulation with Particle Studio PIC solver.}

In the technical note [1] three models of the FNAL RFQ were considered. In all three models the RFQ box, stems, and vane modulation are the same; the differences are in the tuner positions and sizes of the attached beam pipes. The RF fields $(E, H)$ of the RFQ working mode calculated by the MWS were described in [1]. They can be imported into the PS, where one can scale the MWS field solution so that it gives the required inter-vane voltage, $V_{0}=72 \mathrm{kV}$.

The PIC solver in the CST PS, in addition to a few built-in particle distribution types, allows external particle distributions to be imported. An external (macro-)particle distribution is prepared as a text file (*.pit) with the number of lines equal to the particle number. Each line contains 10 particle parameters: coordinates $x, y, z$, momenta $(\beta \gamma)_{\mathrm{x}},(\beta \gamma)_{\mathrm{y}},(\beta \gamma)_{\mathrm{z}}$, particle mass, charge, macro-particle charge, and time. It is convenient to choose particles being injected in a fixed plane transverse to their direction of motion, e.g. $x=x_{\min }$ for particles moving in the positive $x$ direction, at different injection times. The macroparticle charge $q$ is defined by the beam current $I$ and chosen number $n$ of macro-particles. In the case of an RFQ working at frequency $f$ with $\mathrm{CW}$ beam injection, $q=(I / f) / n$.

One simple initial distribution for checking the RFQ output energy is a set of particles at a fixed energy injected along the same line (e.g., on axis) and distributed evenly in time within one RF period $T=1 / f=$ $4.97 \mathrm{~ns}$ for $f=201.25 \mathrm{MHz}$, i.e. particles injected at times $0, T / n, 2 T / n, \ldots,(n-1) T / n$. Such a linear distribution allows one to include all initial RF phases in determining the average output energy. For an energy check, it is also convenient to set the macro-particle charge very low to eliminate space-charge effects.

More realistic initial distributions should be matched to the RFQ for a design current. Such distributions can be generated by beam dynamics codes, e.g. Parmila [5]. Using matched beam parameters from the FNAL RFQ design documents [6], Larry Rybarcyk generated such a distribution of $\mathrm{H}^{-}$ions for $60 \mathrm{~mA}$ at $35 \mathrm{keV}$ [7]. This is a CW distribution so the particles are evenly distributed in phase within one RF period. One should note that the matched parameters [6] correspond to a particular point in the RFQ, the beginning of the vanes. The particles from that point have to be traced back to the beginning of the entrance beam pipe, which was accomplished again with Parmila [5]. Using a simple Matlab script, the Parmila-generated distribution was reformatted into the PS input format (*.pit) described above, 
repeated for $N$ RF periods, and shifted in space, so that the macro-particles are injected in the RFQ at $x$ $=x_{\min }$ within the time interval $[0, N T]$.

The CST Particle Studio PIC solver is a general PIC code. Unlike specialized beam dynamics codes [5], it does not have built-in diagnostics of beam parameters like rms size, emittance, energy spread, etc. However, in PS one can set particle monitors: in configuration space, 3D monitors record particle positions within simulation volume at specified times and 2D ones record particles crossing a fixed plane within certain time interval, plus there are various phase-space monitors. Information recorded by such monitors can be extracted and post-processed to obtain the usual beam dynamics parameters.

\section{Results.}

As an illustration, Fig. 1 shows macro-particles in the RFQ model A recorded by a 3D monitor at the moment $t=302 \mathrm{~ns}$ after the injection start. The 3D monitor was set to record particles every $1 \mathrm{~ns}$. In this particular PS simulation, a $60-\mathrm{mA} \mathrm{CW} \mathrm{H}^{-}$beam is injected at $35 \mathrm{keV}$ during $N=70 \mathrm{RF}$ periods (total $70 \times 10 \mathrm{~K}$ particles), and at the shown moment the particles fill the whole RFQ length and some already exited the structure. The total number of macro-particles at this moment is about $586 \mathrm{~K}$. The particle energy is indicated by color; the energy scale is in the upper right corner. One can see the injected beam converging to the vane entrance; this convergence should be provided by a low-energy beam transport from the ion source. Figure 1 shows how the injected $\mathrm{CW}$ beam is being bunched in the buncher section and how the emerging bunches are being accelerated to $750 \mathrm{keV}$ in the accelerator section near the RFQ end. Some particles are lost off axis, and some come out of RFQ off energy; the latter ones will likely be lost in the following beam transport.

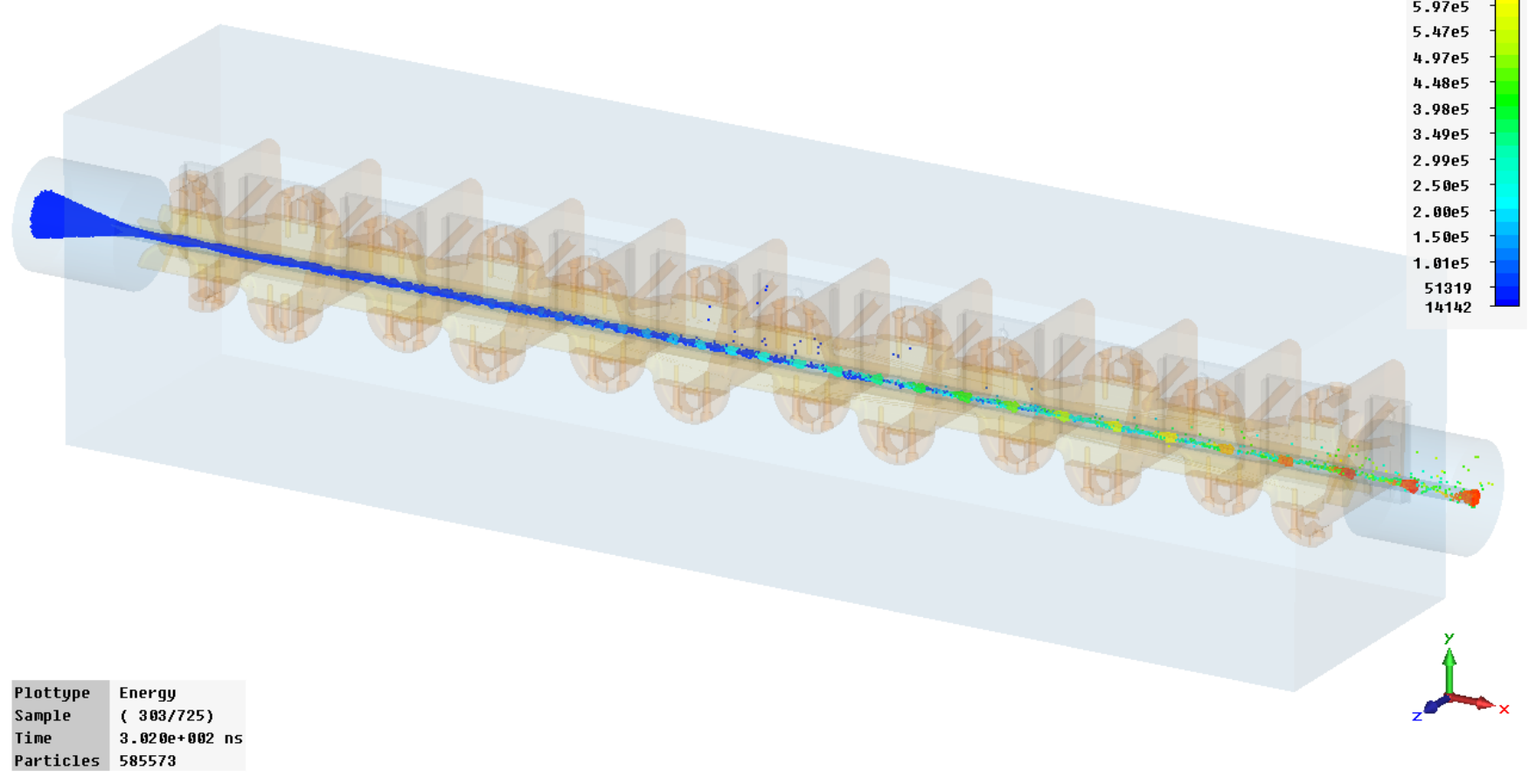

Figure 1: Particles in the RFQ model A at $t=302$ ns. Color indicates particle energy. 
Figure 2 shows the number of macro-particles $N$ in three RFQ models versus time $t$. The number increases almost linearly (without small particle losses it would be exactly linear) till the moment when the first particles reach the RFQ exit: 265-300 ns, depending on the model length. After that there is a flat top for about 80-50 ns during which the $\mathrm{CW}$ beam injection still continues, with small zigzags corresponding to exiting bunches. Then by $\sim 350 \mathrm{~ns}$ the injection is completed, and the number of particles starts to decrease; small steps on the falling slop are due to the exiting bunches. The total number of exiting bunches is slightly more than 70 due to effects of space charge in the longitudinal direction that push particles at the head and tail of the injected beam into adjacent RF buckets. One can notice a small deviation from linear growth in model $\mathrm{C}$, due to particle losses along the structure.



Figure 2: Number of particles in the RFQ models A, B, and C versus time when a $60-\mathrm{mA} \mathrm{CW} \mathrm{H}^{-}$ $70 \mathrm{x} 10 \mathrm{~K}$ beam is injected along the field symmetry axis.

Another way to look at what happens in the PS simulation is using phase-space snapshots. Figure 3 shows a snapshot of the longitudinal phase space $x-W$ (coordinate-energy) at $t=300 \mathrm{~ns}$. One can see the bunch formation and acceleration along the structure as well as the particles not captured in bunches and falling off energy. In Fig. 4, five subsequent phase-space snapshots, at $t=300,301,302,303,304 \mathrm{ns \text {, }}$ are overlapped. Each one is shown in different color, and together they cover about one RF period. Such phase-space distributions can be exported into text files and post-processed to determine the beam parameters like bunch energy and coordinate distribution. However, even qualitative comparison of phase-space snapshots from different models can provide useful information. For instance, Fig. 5 shows two pictures of the longitudinal phase space near the RFQ exit in models A and B. The top picture is a detail of Fig. 4 (its upper-right corner) for model A, and the bottom one is the same for model B: 5 overlapped snapshots separated by $1 \mathrm{~ns}$ starting at $t=301 \mathrm{~ns}$. One should recall that in both models the vane modulation ends at $x \approx 570 \mathrm{~mm}$, the vanes themselves end at $x=588.7 \mathrm{~mm}$, and the RFQ box end wall is at $x=600 \mathrm{~mm}$, see in [1]. The bunch energy increases as it moves to about $x=570 \mathrm{~mm}$ and stays the same till the vane end, then changes somewhat in the end gap ( $x$ from 588.7 to $600 \mathrm{~mm}$ ) and the exit beam pipe $(x>600 \mathrm{~mm})$, due to the longitudinal electric end-gap field, see Figs. 12-13 in [1]. 
583109 particles@ 300 ns

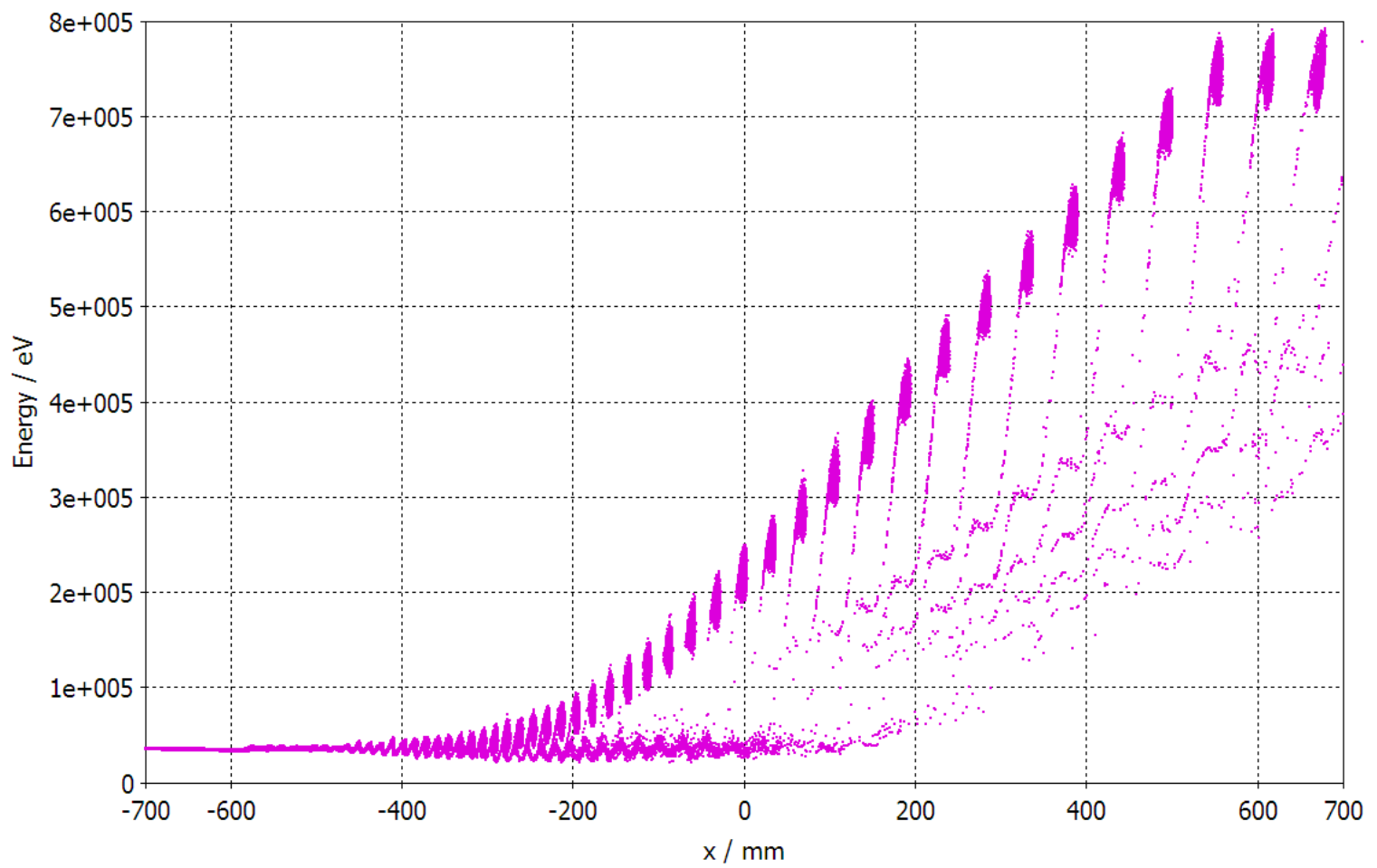

Figure 3: Longitudinal phase space in the RFQ model A at $t=300 \mathrm{~ns}$.

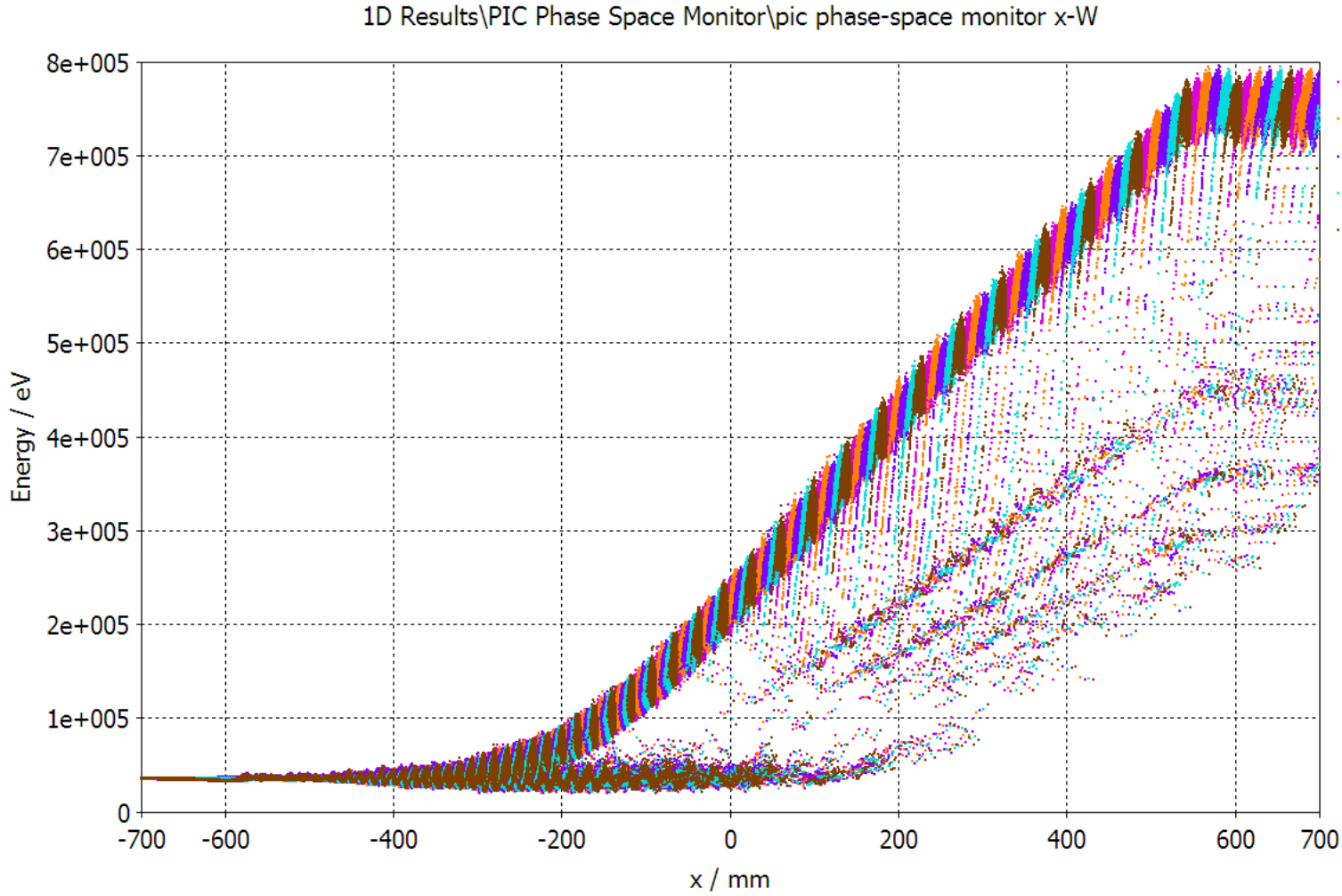

Figure 4: Five overlapping snapshots of the phase space with 1-ns step starting at $t=300 \mathrm{~ns}$. 
1D Results \PIC Phase Space Monitor $\backslash$ pic phase-space monitor $x-W$



1D Results\PIC Phase Space Monitor|pic phase space monitor 1

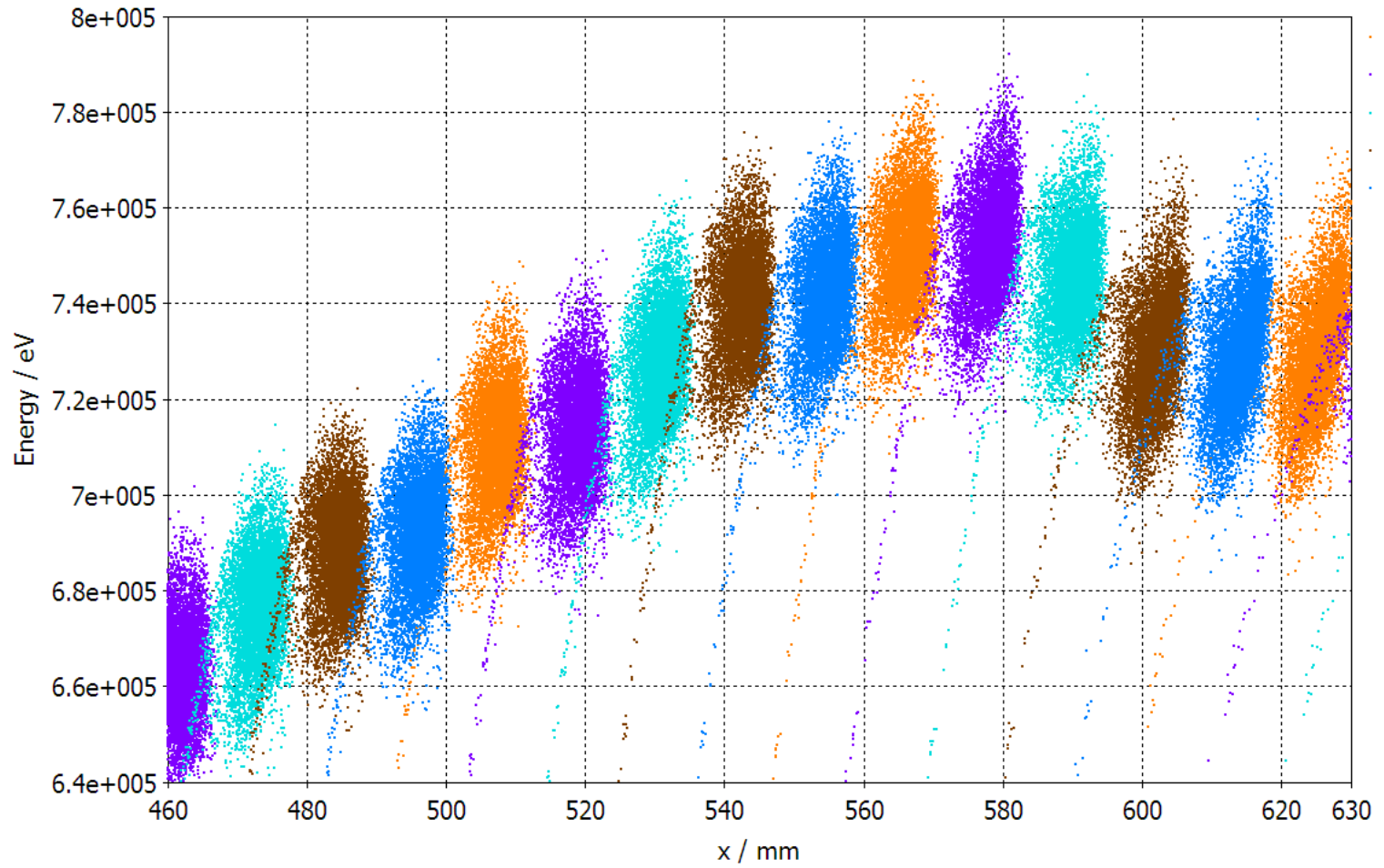

Figure 5: Comparison of five overlapping snapshots of the phase space with 1-ns step near the RFQ exit in model A (top; this is detail of Fig. 4) and in model B (bottom).

One can notice that the energy change near the RFQ exit in model A is rather small, and the bunch average energy is close to the design value, $750 \mathrm{keV}$. In contrast, in the RFQ model B the energy drops 
noticeably, to about $725 \mathrm{keV}$, which agrees with our estimates described in the end of Sec. 3 in [1]. It is worth reminding that the two models have different attached beam pipes: $30-\mathrm{mm}$ long pipes of $15-\mathrm{mm}$ diameter in model $\mathrm{B}$, and $100-\mathrm{mm}$ long ones of $100-\mathrm{mm}$ diameter in $\mathrm{A}$. This is the reason why the longitudinal scales in Figs. 5 are different: model A extends to $x=700 \mathrm{~mm}(600+100)$ but $\mathrm{B}$ only to $x=$ $630 \mathrm{~mm}(600+30)$.

Before we proceed with quantitative results from 2D monitors, one should mention that Fig. 1 may create a misleading impression that the number of particles outside well-formed bunches near the RFQ end is significant. This is because single particles outside of a bunch are plotted by single points that are visible but points for multiple particles packed inside the bunch are overlapping. Figure 6 gives a closeup view of a few bunches near the RFQ exit in a different PS run of model A with 60x10K particles injected. It is important to emphasize here that the number of particles lumped in bunches (red blobs), about 9000 particles per bunch, is significantly larger than between them. The bunch spacing at $\beta=0.04$ $(750 \mathrm{keV})$ is $\beta \lambda=6 \mathrm{~cm}$, where $\lambda=149 \mathrm{~cm}$ is the RF wavelength, cf. the last 5 bunches in Fig. 3 . The blue channel-like structure in Fig. 6 where bunches travel is the vacuum insert of $10 \mathrm{~mm} \times 10 \mathrm{~mm}$ cross section along the RFQ axis that was used in MWS calculations [1] as an artificial feature to make the mesh denser in and around the RFQ aperture.



Figure 6: Particles in the RFQ model A at $t=297$ ns with 60x10K beam: close-up near exit. Color indicates particle energy.

The PS 2D particle monitors record particles crossing a fixed plane within specified time intervals. The recorded information can be extracted into a *.pit file that may be used either for post-processing or as an input for follow-up simulations. To evaluate the RFQ exit-beam parameters, we set $2 \mathrm{D}$ monitors in the exit beam pipe - in a transverse plane at $x=650 \mathrm{~mm}$ in model $\mathrm{A}$, and at $x=620 \mathrm{~mm}$ in model B with transverse boundaries $-10 \mathrm{~mm}<y, z<10 \mathrm{~mm}$ and a short time step of $0.1 \mathrm{~ns}$. One snapshot of the 
2D monitor in model A is illustrated in Fig. 7. At the moment $t=303.1 \mathrm{~ns}$, the monitor recorded 1212 particles within $0.1 \mathrm{~ns}$, mostly at or near the design energy of $750 \mathrm{keV}$. This is a $0.1-\mathrm{ns}$ slice of a bunch. Between bunches the number of particles recorded within 0.1 ns varies from 0 to a few, and most of them have lower energies, cf. Fig. 6.

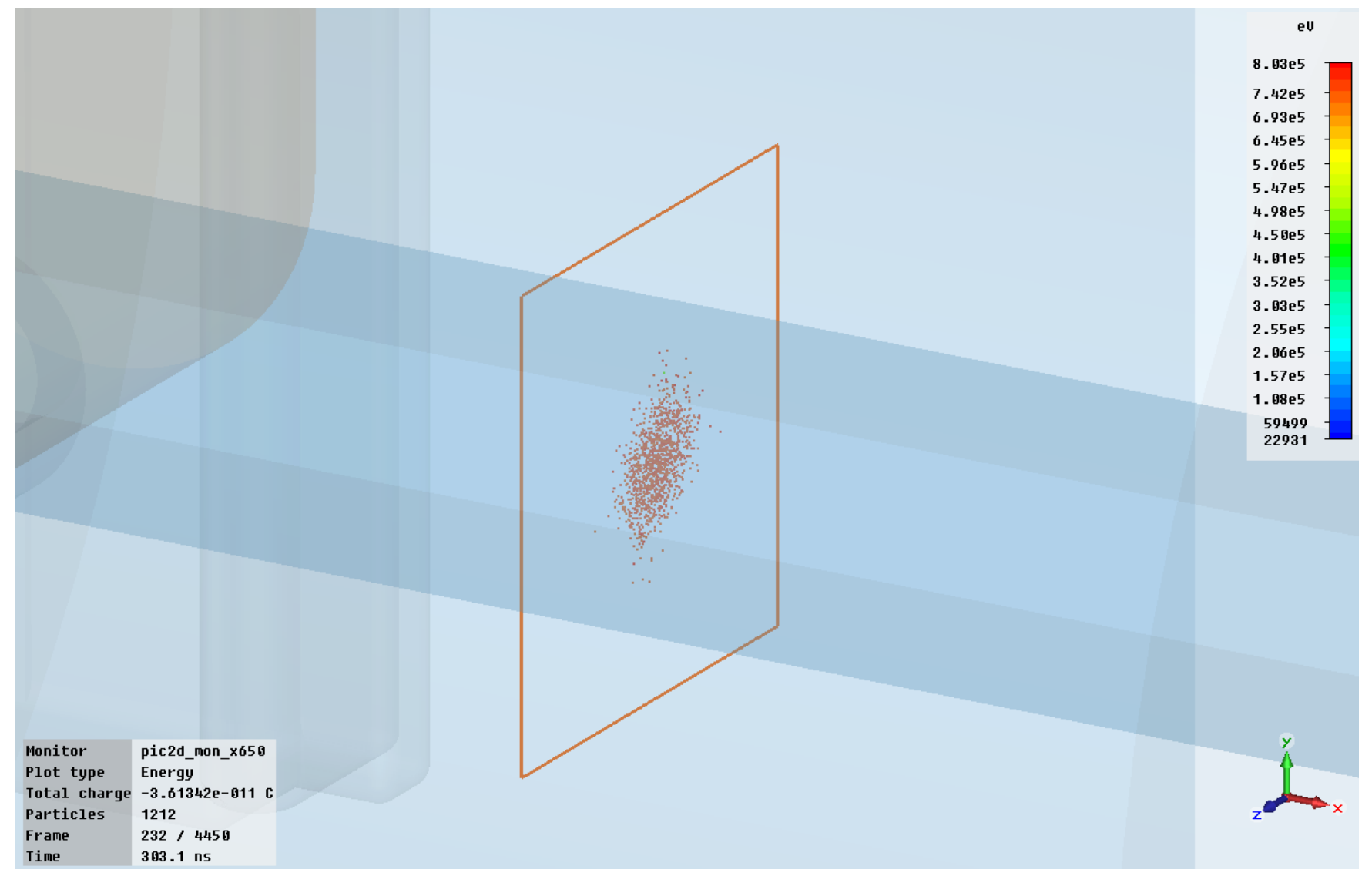

Figure 7: Particles recorded by 2D monitor (red square) at $x=650 \mathrm{~mm}$ in the RFQ model A at $t=303.1$ ns within $0.1 \mathrm{~ns}$ during PS run with 70x10K beam. Particle color indicates its energy (see the scale).

Post-processing of 2D PIC monitor results gives more quantitative data. We run a Matlab script that reads and manipulates the data in a *.pit file. As an example of such post-processing, Fig. 8 plots the energy of exiting particles recorded by a 2D monitor at $x=620 \mathrm{~mm}$ from PS simulation of the RFQ model B with a matched $\mathrm{CW} 35-\mathrm{keV}$ input $\mathrm{H}^{-}$beam at $60 \mathrm{~mA}$ injected over $10 \mathrm{RF}$ periods (10x10K). One can see that the exiting particles form not 10 but at least 12 bunches (due to space-charge pushing particles into the adjacent RF buckets). The total number of exiting particles is 89486 and their average energy is $729.1 \mathrm{MeV}$; however, some have significantly lower energies and will be lost downstream. To take the acceptance of the following structures into account, we introduce the energy cut to include only $\pm 50 \mathrm{keV}$ around the average energy. Strictly speaking, it should be $\pm 50 \mathrm{keV}$ cut around the design energy, $750 \mathrm{keV}$. In this case, just as an example, we select particles with $W>680 \mathrm{keV}$; there are 87563 such particles, which means that only $2.2 \%$ of particles in Fig. 8 are below $680 \mathrm{keV}$. In addition, we only choose 6 central bunches as ones less distorted by edge effects. The number on energetic particles $(W>680 \mathrm{keV})$ in 6 central bunches is 52654 , i.e. the RFQ effective transmission rate from this simulation is $52654 / 60000=0.878$. 


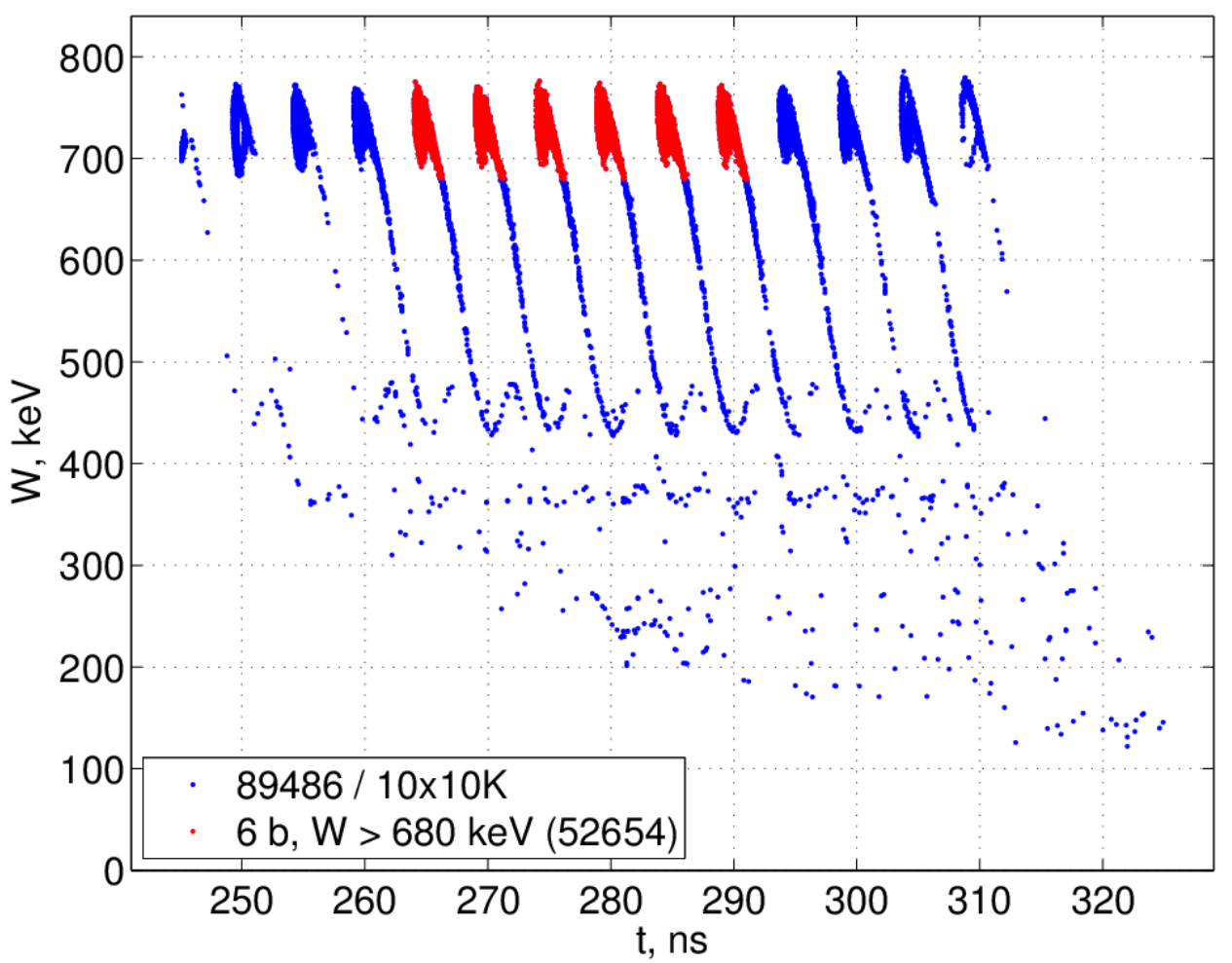

Figure 8: Results from 2D monitor at $x=620 \mathrm{~mm}$ in the RFQ model B with $10 \mathrm{x} 10 \mathrm{~K}$ beam: particle energy versus time (blue - all exiting particles, red -6 central bunches with energy cut below $680 \mathrm{keV}$ ).

Details on bunch population and energy, with and without energy cut, are presented in Figs. 9 and 10. Figure 9 shows the bunch population with all exiting particles included (left, blue), and when only 6 central bunches are considered plus particles with energy below $680 \mathrm{keV}$ are filtered out (right, red). Figure 10 plots the average energy of the exiting bunches. Obviously, it is better to discard a few bunches in the head and tail of the bunch train when calculating the exiting beam parameters.
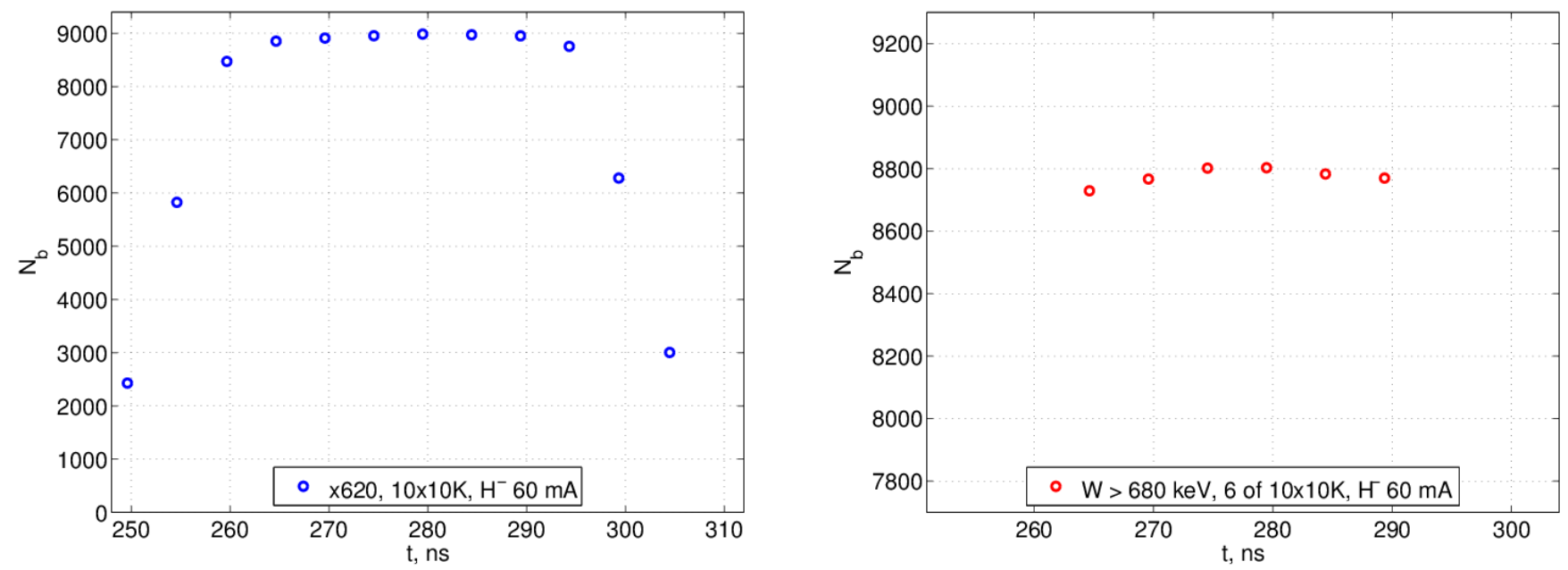

Figure 9: Bunch population versus time from 2D monitor at $x=620 \mathrm{~mm}$ in model B with $10 \mathrm{x} 10 \mathrm{~K}$ beam (blue - all exiting particles, red -6 central bunches with energy cut below $680 \mathrm{keV}$, cf. Fig. 8). 

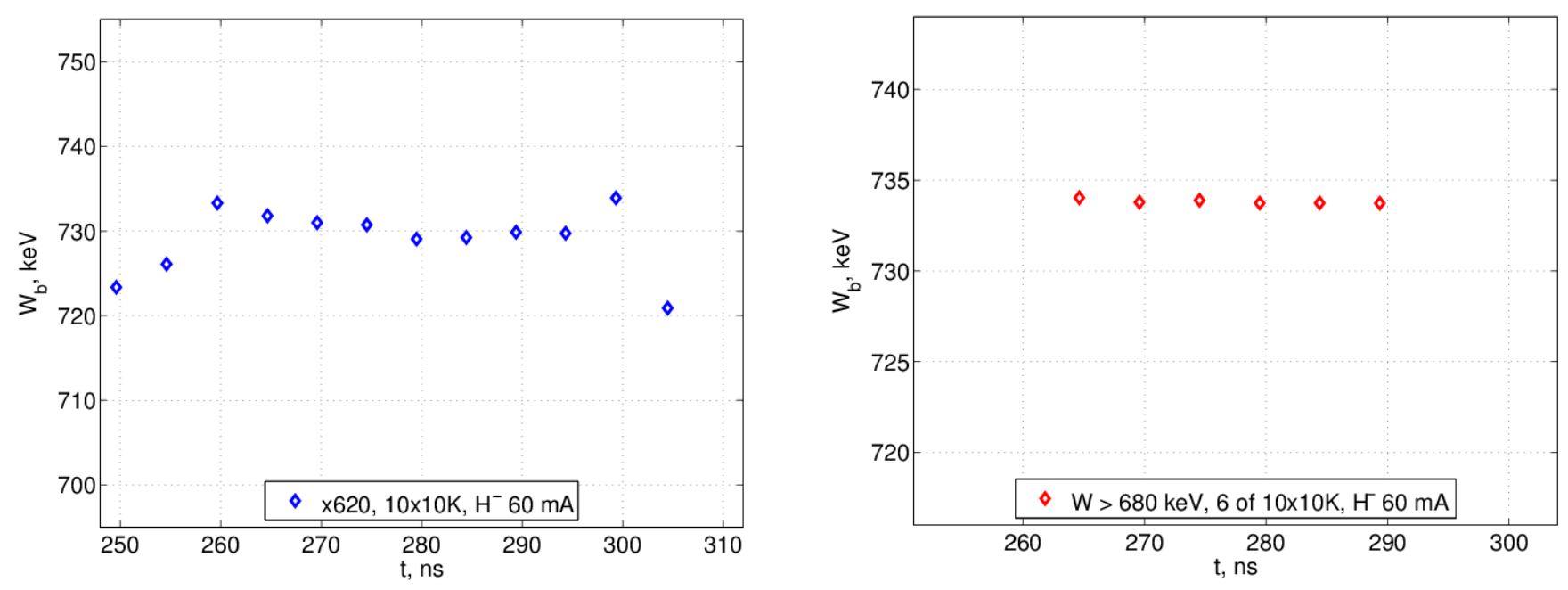

Figure 10: Average energy of exiting bunches from 2D monitor at $x=620 \mathrm{~mm}$ in model B with $10 \mathrm{x} 10 \mathrm{~K}$ beam (blue - all particles, red - 6 central bunches with energy cut below $680 \mathrm{keV}$ ).

In PS simulations for models $\mathrm{A}$ and $\mathrm{B}$ with a matched $\mathrm{CW} 70 \times 10 \mathrm{~K} \mathrm{H}^{-}$beam at $60 \mathrm{~mA}$ injected, we record all particles with 2D PIC monitors described above. Below we consider only 50 central bunches plus apply an energy cut at $\pm 50 \mathrm{keV}$ around the average energy. The results for bunch energy and population for the two models are compared in Figs. 11-12. One can see again from Fig. 11 that the RFQ exit energy is low by $21 \mathrm{keV}$ in model $\mathrm{B}$.
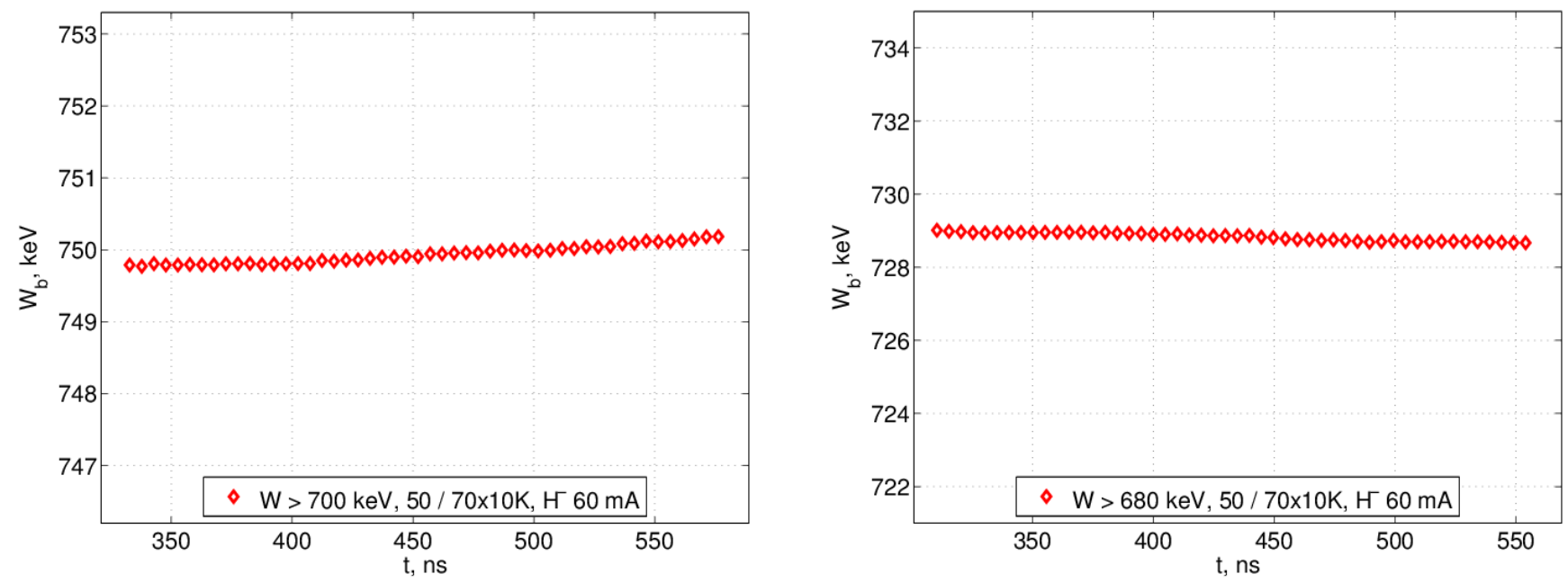

Figure 11: Average bunch energy in the RFQ models A (left) and B (right) beam for 50 central bunches with energy cut (700 keV for A and $680 \mathrm{keV}$ for B) from PS simulations with 70x10K input beam.

Since the maximal possible number of exiting particles per RF period is $10 \mathrm{~K}$ in our PS simulations, assuming no losses and stationary state, the bunch population plots in Fig. 12 give us the RFQ transmission rate. It is $90.5 \%$ in model $\mathrm{A}$ and $92.5 \%$ for $\mathrm{B}$. There is a small drop in the case B from more than $93 \%$ for first central bunches to $92 \%$ for the last ones; it is not clear what can cause such a behavior. 

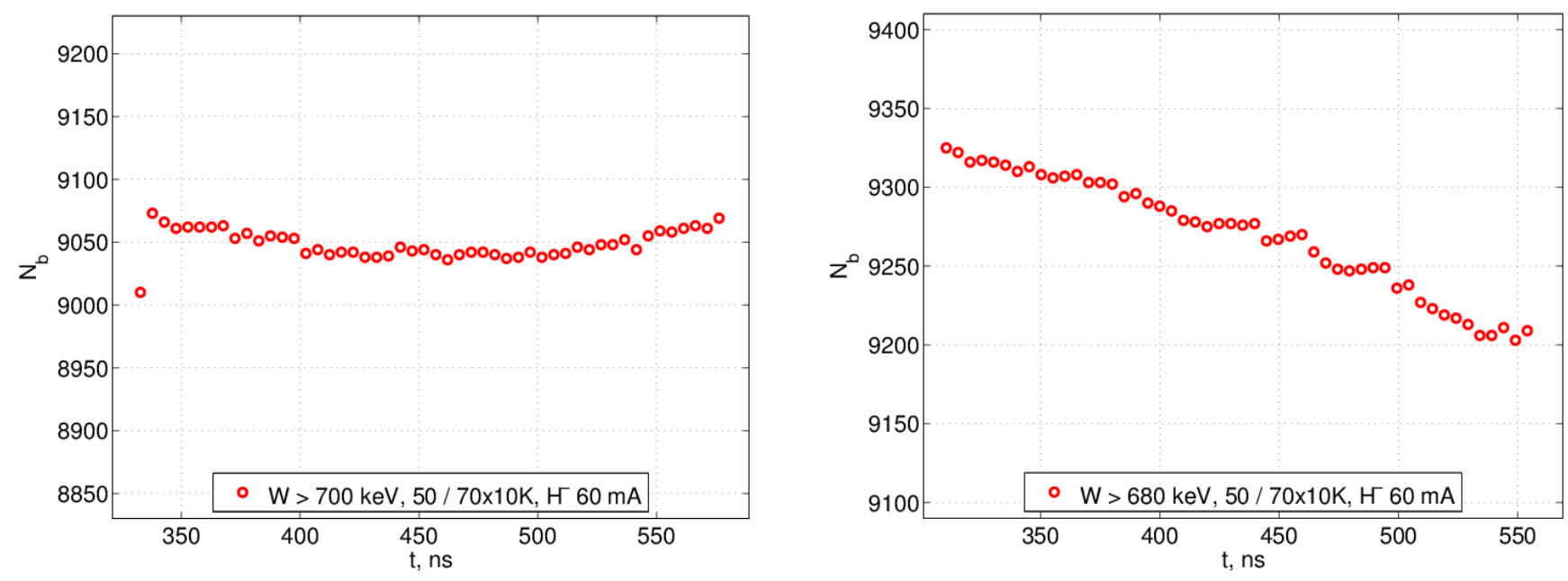

Figure 12: Number of particles in 50 central bunches from PS simulations with 70x10K input beam in the RFQ models A (left) and B (right). The particle low energy cut is $700 \mathrm{keV}$ for A and $680 \mathrm{keV}$ for B.

Figures 13-14 plot the final emittances of the 50 central bunches from the PS simulations of the models $\mathrm{A}$ and $\mathrm{B}$ with $70 \times 10 \mathrm{~K} 60-\mathrm{mA} \mathrm{H}^{-}$beam. The initial transverse normalized rms emittances were chosen to be $0.3 \pi \mathrm{mm} \cdot \mathrm{mrad}$ in both planes. The emittance results from two models are close to each other. One should clarify the notations used in the emittance plots in Fig. 13: the horizontal transverse emittance in the plane parallel to the RFQ ground plate - is denoted $\varepsilon_{\mathrm{y}}$ and corresponds to the coordinate system in Fig. 1, but for the vertical transverse emittance (in the plane along the stems) we used $\varepsilon_{\mathrm{x}}$, though it would be $\varepsilon_{z}$ if we followed Fig. 1, where $x$ is the longitudinal coordinate.
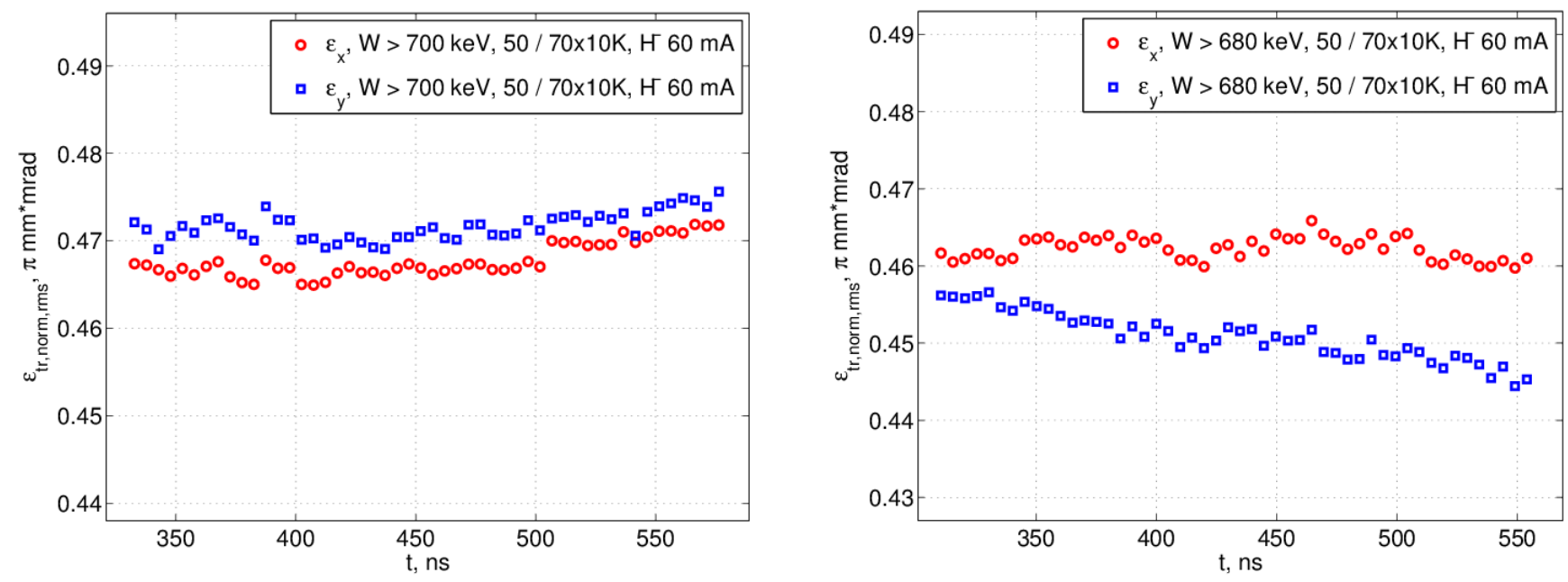

Figure 13: Transverse normalized rms emittances in the RFQ models A (left) and B (right) beam for 50 central bunches with energy cuts from PS simulations with 70x10K input beam.

One should mention that for the PS simulations described above, the initial particle distributions were injected centered on the field symmetry axis, which is parallel to the RFQ geometrical axis but displaced from it by $-0.5 \mathrm{~mm}$ in $z$ (vertically, along the stems), see discussion in [1]. For comparison, we also studied one case for the model A when the initial $70 \times 10 \mathrm{~K}$ beam was centered on the geometrical axis. The results are close to those described above, except that the transmission is reduced by about $2-3 \%$. The horizontal transverse emittance $\left(\varepsilon_{\mathrm{y}}\right)$ values are the same as in Fig. 13 (left), but $\varepsilon_{\mathrm{x}}$ is a bit higher, about $0.48 \pi \mathrm{mm} \cdot \mathrm{mrad}$. 

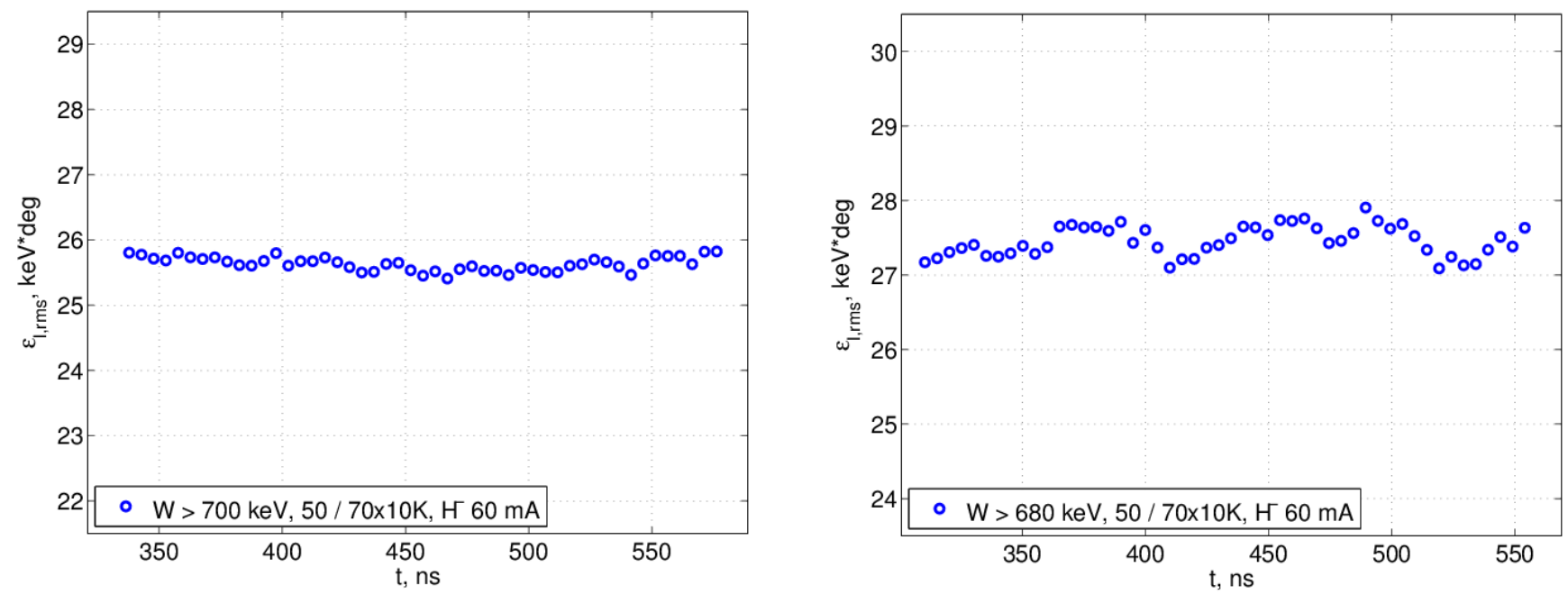

Figure 14: Longitudinal rms emittances in the RFQ models A (left) and B (right) beam for 50 central bunches with energy cuts from PS simulations with 70x10K input beam.

In the process of the FNAL RFQ commissioning for production it was found [8] that up to $10 \mathrm{~mA}$ were being lost after the RFQ in a short Medium-Energy Beam Transfer (MEBT) to DTL. The problem was traced to the beam exiting RFQ at an angle of $1-2^{\circ}$ vertically (in the lab frame). We used the existing data from PS 2D monitors to check if such an effect was observed in simulations. Figure 15 shows the angular distributions of the exiting particles in the RFQ model A in the vertical and horizontal planes. The RFQ is installed at FNAL rotated by $45^{\circ}$ in the transverse plane around its longitudinal axis compared to Fig. 1 so that both $y$ and $z$ vectors in Fig. 1 are at $45^{\circ}$ above the horizontal lab plane $(h)$, and the vertical positive direction $(v)$ is between them. One can see that the horizontal angle distribution is almost symmetric around zero, with the average value $<\theta_{\mathrm{h}}>=0.2^{\circ}$, but the vertical angles are skewed down, with $<\theta_{\mathrm{v}}>=-0.5^{\circ}$.
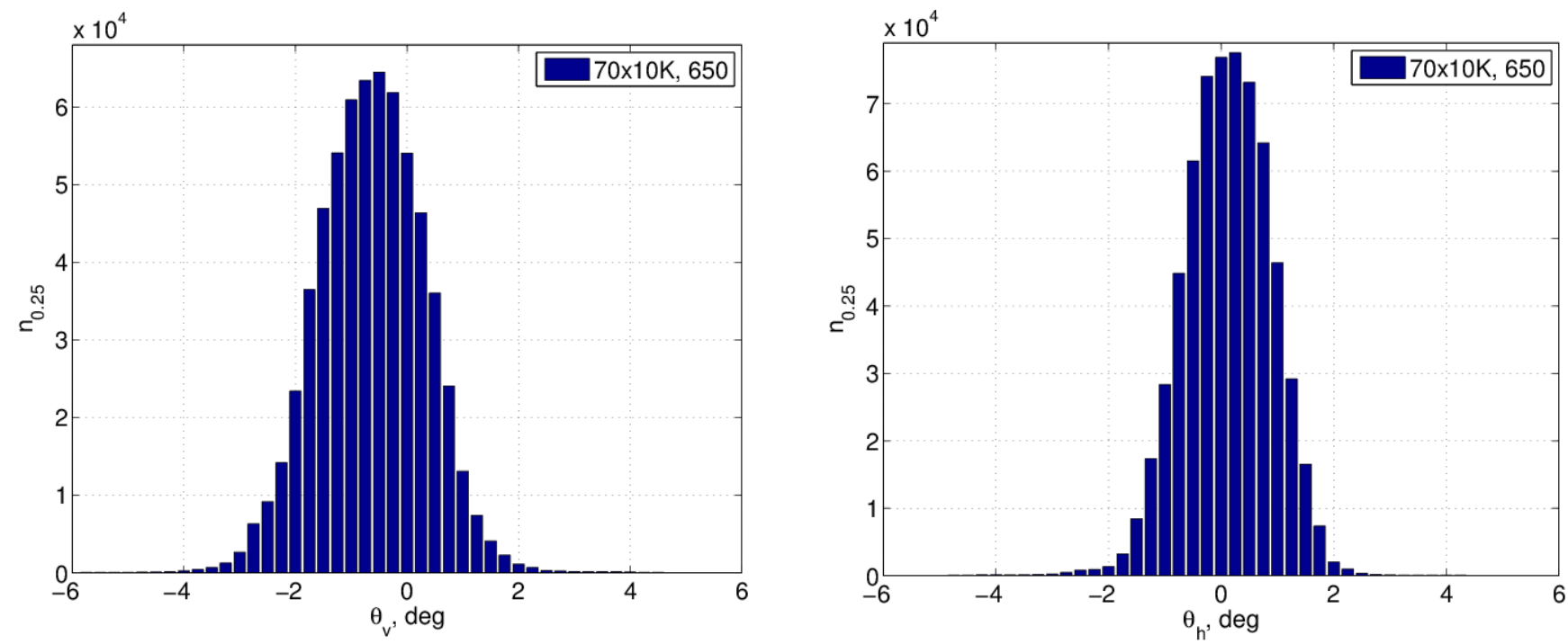

Figure 15: Histograms of particle exit angles in the RFQ model A - vertical (left) and horizontal (right) from 2D monitor at $x=650 \mathrm{~mm}$ in PS simulations with $70 \times 10 \mathrm{~K}$ input beam. 
From a similar PS run with the initial beam centered on the RFQ geometrical axis, not its field axis, the average exit vertical angle is even more negative, $\left\langle\theta_{\mathrm{v}}>=-0.8^{\circ}\right.$. Moreover, the vertical beam positions in the monitor plane, $x=650 \mathrm{~mm}$, are also shifted down, with the average value $\langle v>=-1.2 \mathrm{~mm}$, see in Fig. 16.
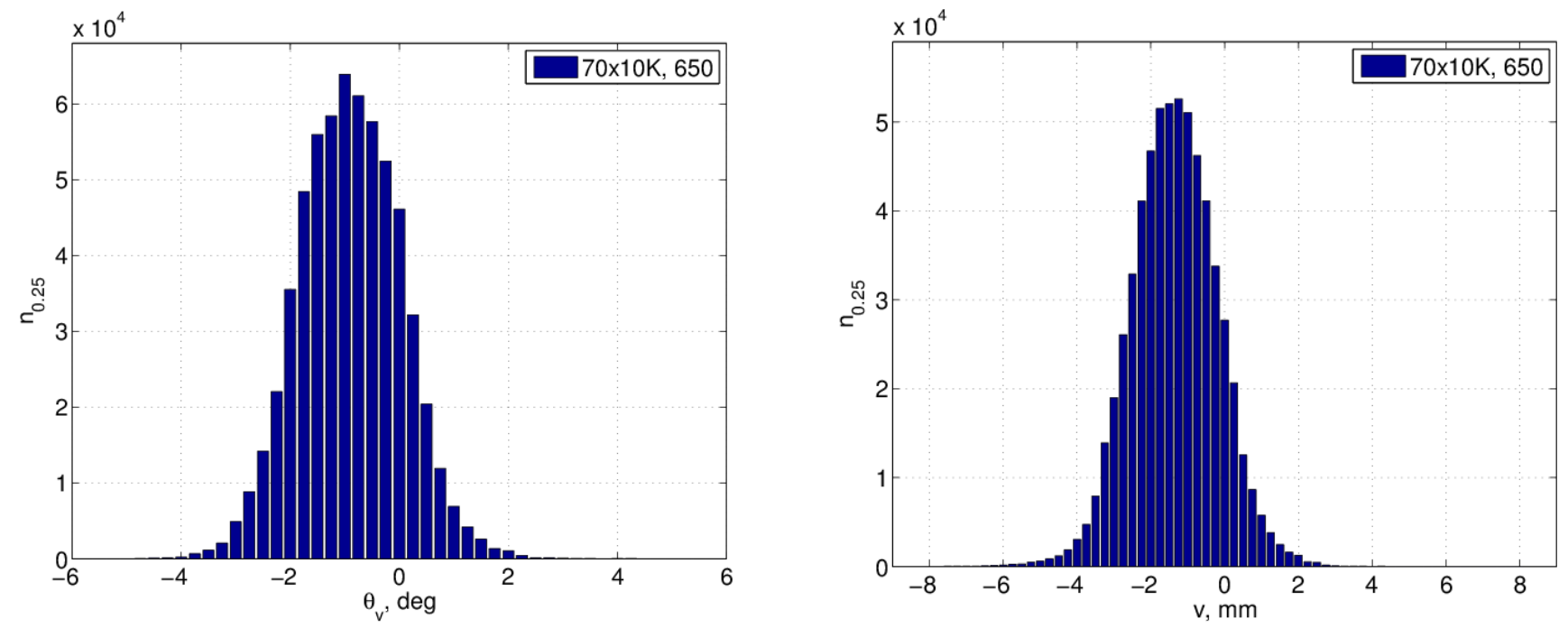

Figure 16: Histograms of particle vertical exit angle (left) and vertical position (right) in the model A at $x=650 \mathrm{~mm}$ from PS simulations with $70 \times 10 \mathrm{~K}$ input beam centered on the RFQ geometrical axis.

It is also interesting to see how these results depend on the RFQ inter-vane voltage. All PS results above were obtained with the field normalized to the design inter-vane voltage, $V_{0}=72 \mathrm{kV}$. However, most of the time the FNAL RFQ is operated with higher RF input power, i.e. at higher inter-vane voltage. We used results from earlier PS simulations with a linear low-charge particle distribution of 1000 particles evenly spread in phase within one RF period and injected along the field axis in model A, first at the nominal voltage $V_{0}$, and then at $V=1.25 V_{0}$. The exit angular distribution are plotted in Fig. 17; the average vertical exit angle is $\left\langle\theta_{\mathrm{v}}>=-0.4^{\circ}\right.$ at $V=V_{0}$ but becomes $\left\langle\theta_{\mathrm{v}}\right\rangle=-1.1^{\circ}$ for $V=1.25 V_{0}$. The average horizontal angle is small in both cases, $0.1-0.2^{\circ}$.
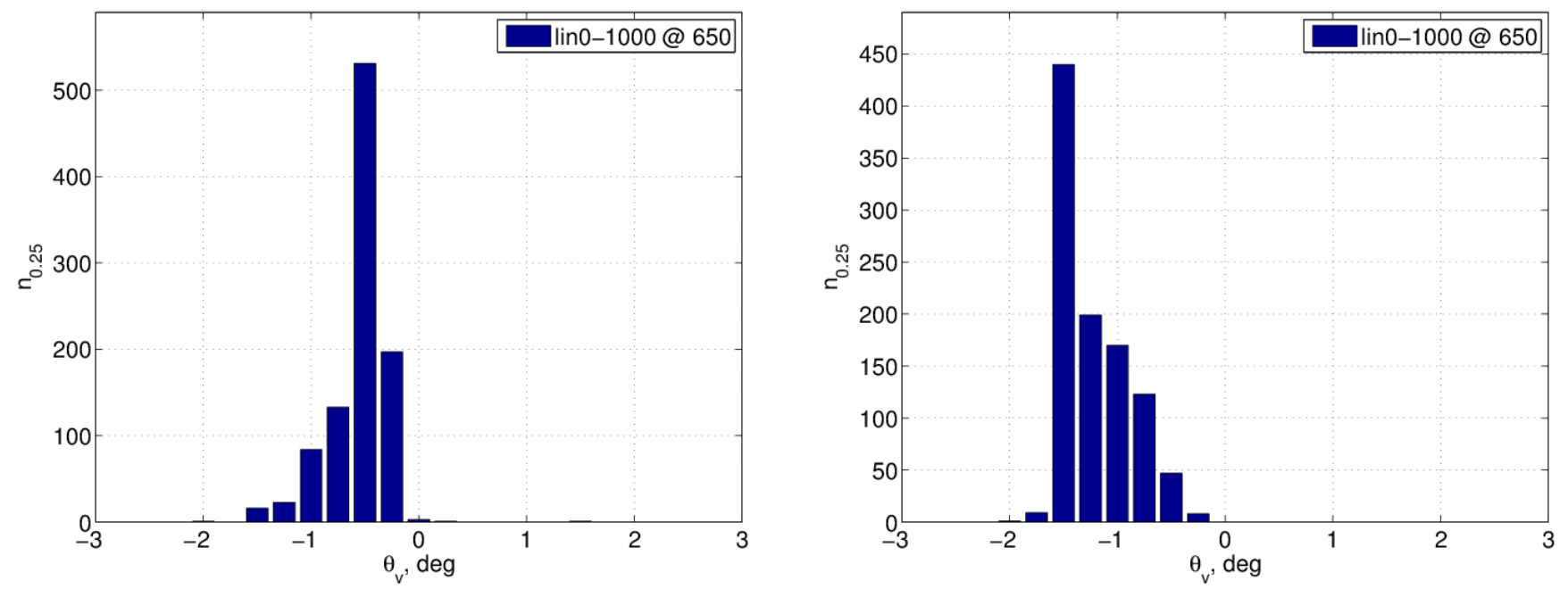

Figure 17: Histograms of particle vertical exit angle at $V=V_{0}$ (left) and $V=1.25 V_{0}$ (right) in the model A at $x=650 \mathrm{~mm}$ from PS simulations with 1000 particle linear input beam at very low current. 
Figure 18 shows exit angle distributions in the RFQ model A with an increased inter-vane voltage, $V=$ $1.25 V_{0}$. The horizontal angle distribution remains symmetric with the average value $<\theta_{\mathrm{h}}>=-0.02^{\circ}$; the vertical angles become more negative, with $\left\langle\theta_{\mathrm{v}}\right\rangle=-1.13^{\circ}$.
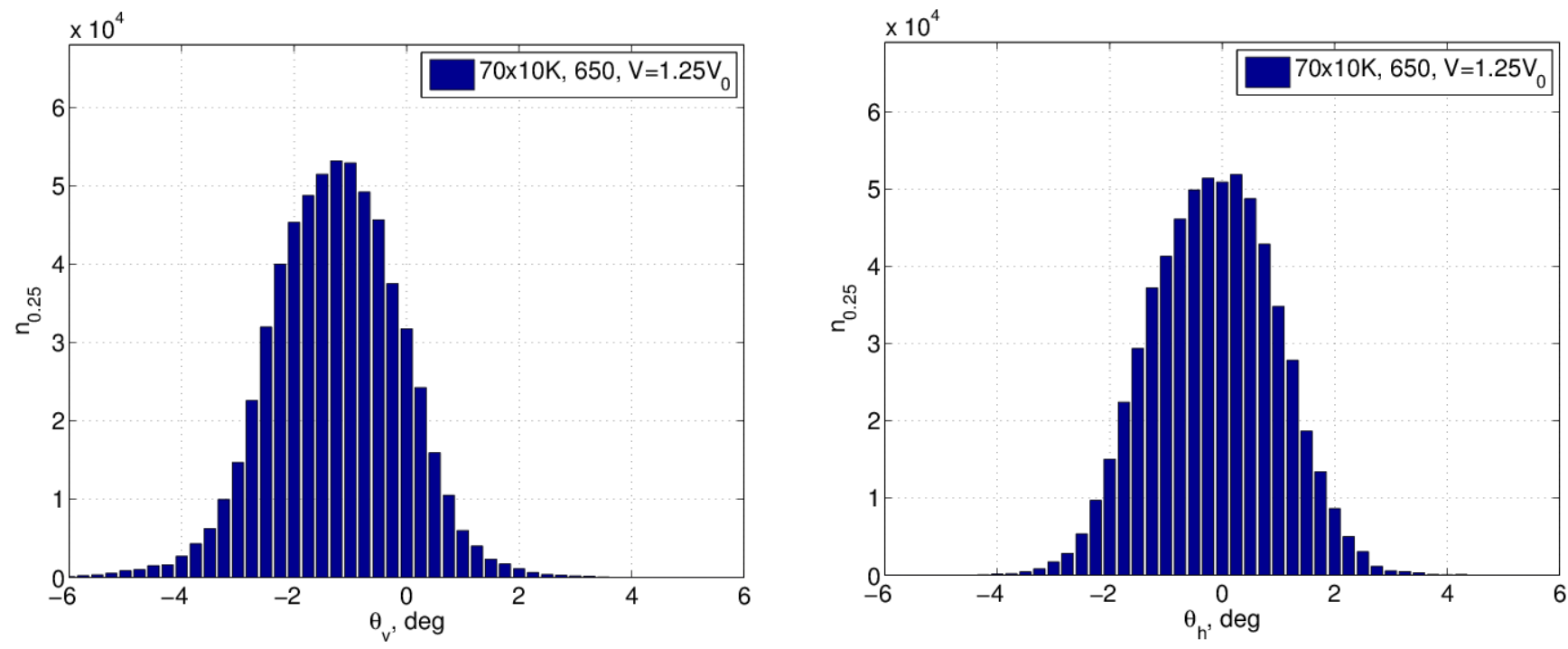

Figure 18: Histograms of particle exit angles in the RFQ model A with $V=1.25 V_{0}$ - vertical (left) and horizontal (right) from 2D monitor at $x=650 \mathrm{~mm}$ in PS simulations with 70x10K input beam.

The exit angle results for the RFQ model B are similar. The average vertical exit angle is $<\theta_{\mathrm{v}}>=-0.8^{\circ}$ at the nominal voltage and injection along the field axis, and $-0.84^{\circ}$ with the increased voltage, $V=1.25 V_{0}$. It becomes $\left\langle\theta_{\mathrm{v}}>=-1.24^{\circ}\right.$ when $V=1.25 V_{0}$ and the beam is injected centered on the RFQ geometrical axis. These and the above results, shown in Figs. 15-18, are consistent with an assumption that the exitgap fields contribute to non-zero exit angles.

It is interesting to see whether the increased inter-vane voltage influences the exit energy of the RFQ beam. Some comparison for the beam energy and transmission from PS runs with 70x10K particles in models A and B are summarized in Tab. 1.

Table 1: Results for 50 central bunches in RFQ models A and B

\begin{tabular}{|l|c|c|c|c|}
\hline Quantity & \multicolumn{2}{|c|}{ Average energy, keV } & \multicolumn{2}{c|}{ Transmission } \\
\hline RFQ model & A & B & A & 0.91 \\
\hline$V=V_{0}$ & 750 & 729 & 0.89 & 0.93 \\
\hline$V=1.25 V_{0}$ & 753 & 729 & & 0.97 \\
\hline
\end{tabular}

One can see that the voltage effect on the exit energy is rather small. It can be explained by the fact that the final energy is defined by both an accelerating effect of the last one-two cells and the exit-gap-field effect. While the energy drop due to the increased field in the exit gap increases, so does the energy gain in the last cells of the RFQ. We illustrate this by two snapshots of a few exiting bunches in the RFQ model B in Fig. 19. The top picture corresponds to the nominal voltage, and the bottom one to the increased voltage, $V=1.25 V_{0}$. Notice the higher maximal particle energy on the energy scale in the bottom picture. One can also see that there are much less off-energy particles between bunches in that case, which is reflected in higher transmission in Tab. 1, likely due to better bunching at higher fields. 

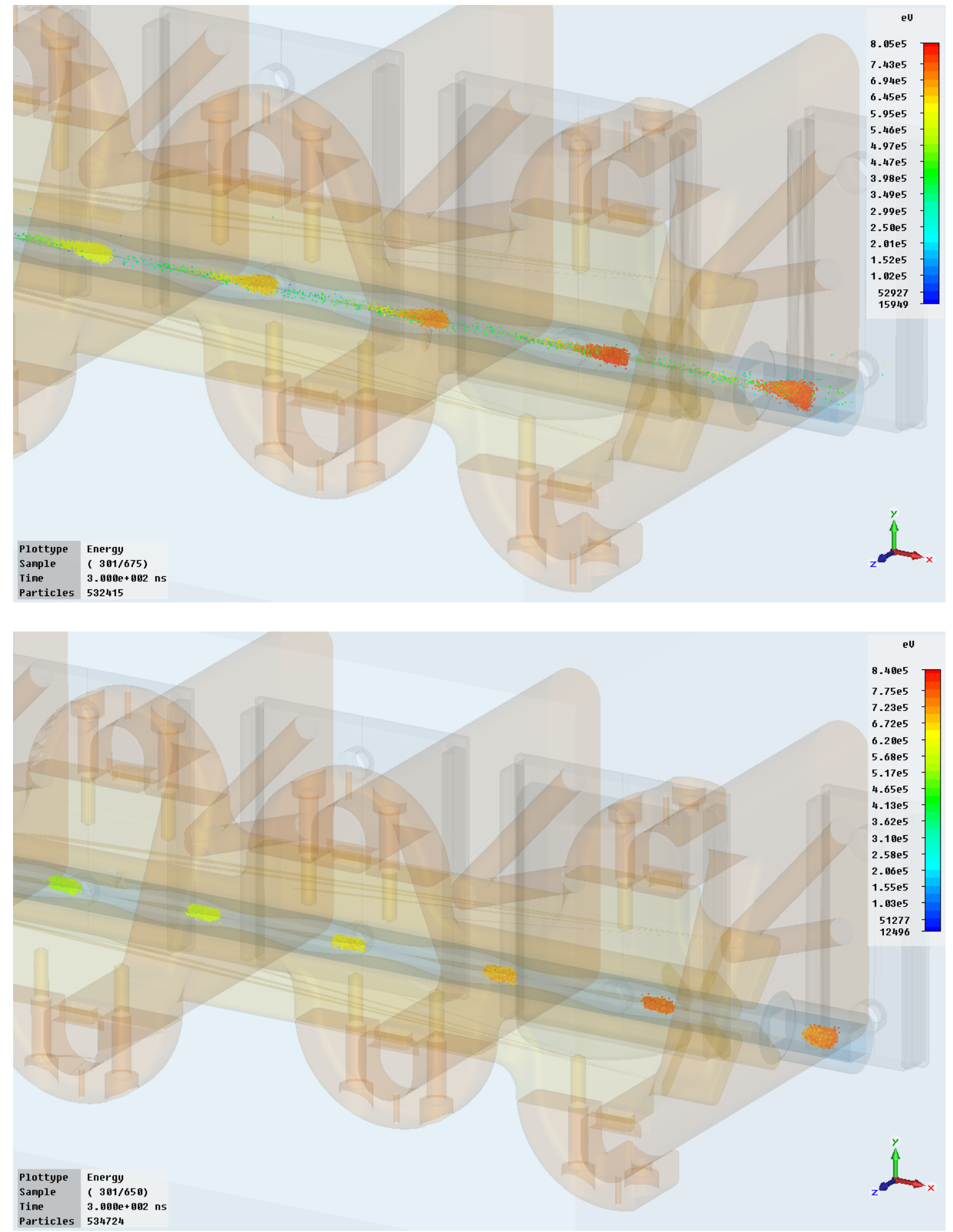

Figure 19: Particles near the exit in the RFQ model B at $t=300 \mathrm{~ns}$ with $70 \times 10 \mathrm{~K}$ beam at the nominal inter-vane voltage $V_{0}$ (top) and the increased one $V=1.25 V_{0}$ (bottom). Color indicates particle energy.

This energy change can also be seen in the longitudinal phase-space plots similar to those in Fig. 5. We plot overlapping snapshots of the longitudinal phase-space with 1-ns step starting at $t=300$ ns near the RFQ exit in model B with $V=1.25 V_{0}$ in Fig. 20. This picture should be compared to one in Fig. 5 
(bottom), where a similar plot was presented for the nominal voltage, $V=V_{0}$. One can see that the bunches near the end of the vane modulation, $x \approx 570 \mathrm{~mm}$, in Fig. 20 have higher energy than in Fig. 5, but the energy drop in the exit gap is also larger. In addition, one can notice that the bunch longitudinal separation is cleaner in Fig. 20 compared to Fig. 5, in agreement with comparison of these two cases in Fig. 19.

1D Results \PIC Phase Space Monitor\pic phase space monitor 1

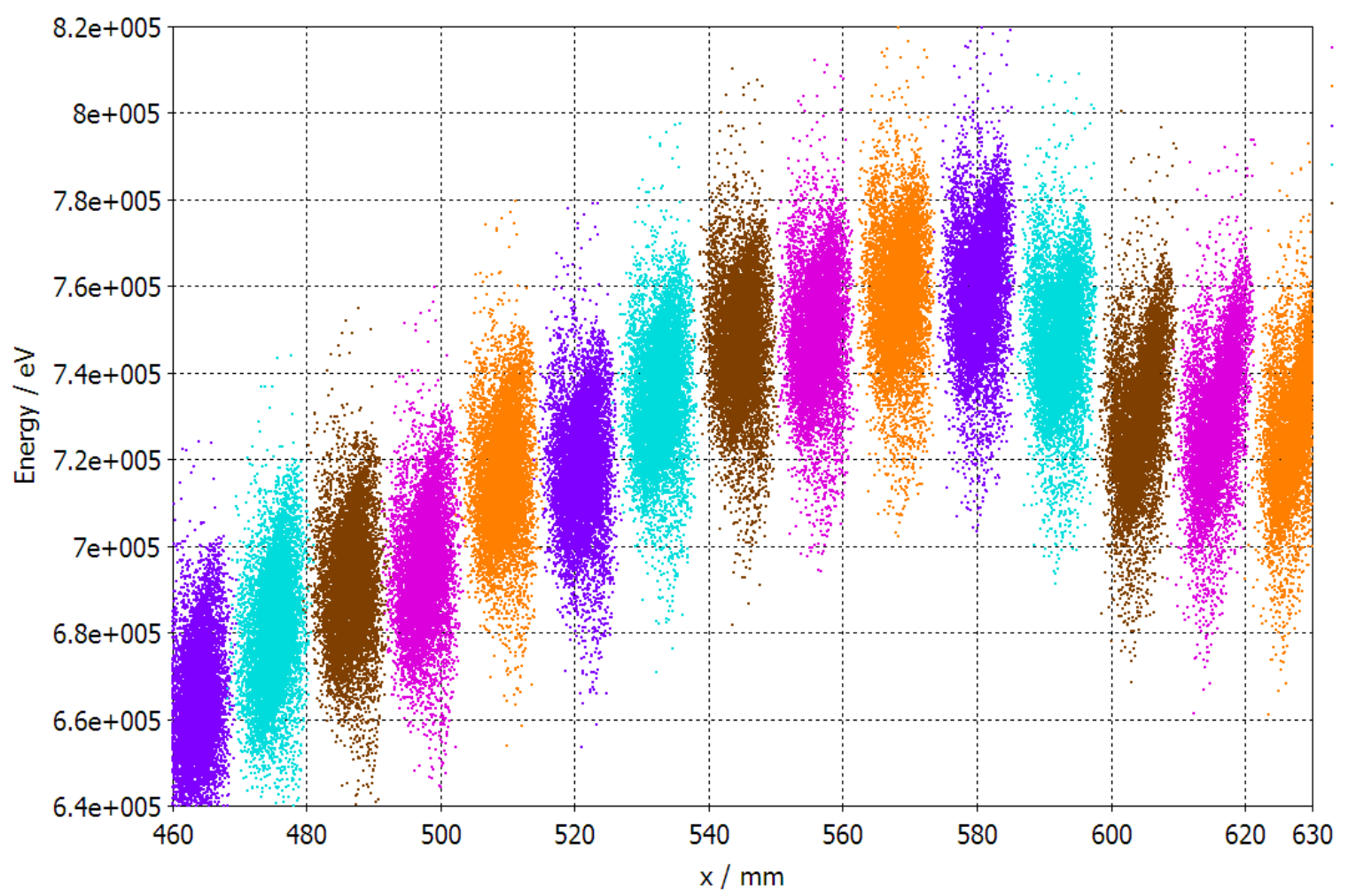

Figure 20: Five overlapping snapshots of the longitudinal phase-space with 1-ns step starting at $t=300$ ns near the RFQ exit in model B with $V=1.25 V_{0}$; cf. Fig.5 (bottom) where $V=V_{0}$.

The beam transmission through an RFQ depends on many factors. We looked first at effects of the voltage profile along the RFQ using the same initial matched beam by comparing two cases: model B and model $B$ with a retuned voltage profile (V3), which was changed from a relatively flat profile in model B (with a 3\% hump near the middle) to resemble more the measured U-shaped voltage profile in the FNAL RFQ, where the end voltages were $\sim 7 \%$ above the average. For the same average inter-vane voltage $V_{0}=72 \mathrm{kV}$, the transmission in the $\mathrm{V} 3$ case is reduced by about $10 \%$ compared to that in the model $\mathrm{B}$ with the flat profile, becoming 0.85 in a $15 \times 10 \mathrm{~K}$ run $(5$ central bunches $)$ or 0.83 in a $70 \times 10 \mathrm{~K}$ run (50 central bunches), cf. Tab. 1. At the same time, the bunch average energy increases to $737 \mathrm{keV}$. However, this reduction in transmission is rather small compared to the cases when initial beam distributions are mismatched. Figure 21 illustrates this conclusion by plotting the number of particles versus time in a few simulations with $15 \times 10 \mathrm{~K}$ injected distributions. The two cases above, B and V3, look very close in Fig. 21, much better than two other cases where the particle losses along the structure are very noticeable. Note that the flat top in the first two cases also slightly deviates from horizontal (the number of particles decreases) at larger times, above $200 \mathrm{~ns}$, due to small beam losses. The latter two cases correspond to our (likely incorrect) interpretation of the FNAL measurements of beam parameters at the RFQ entrance [9]. 


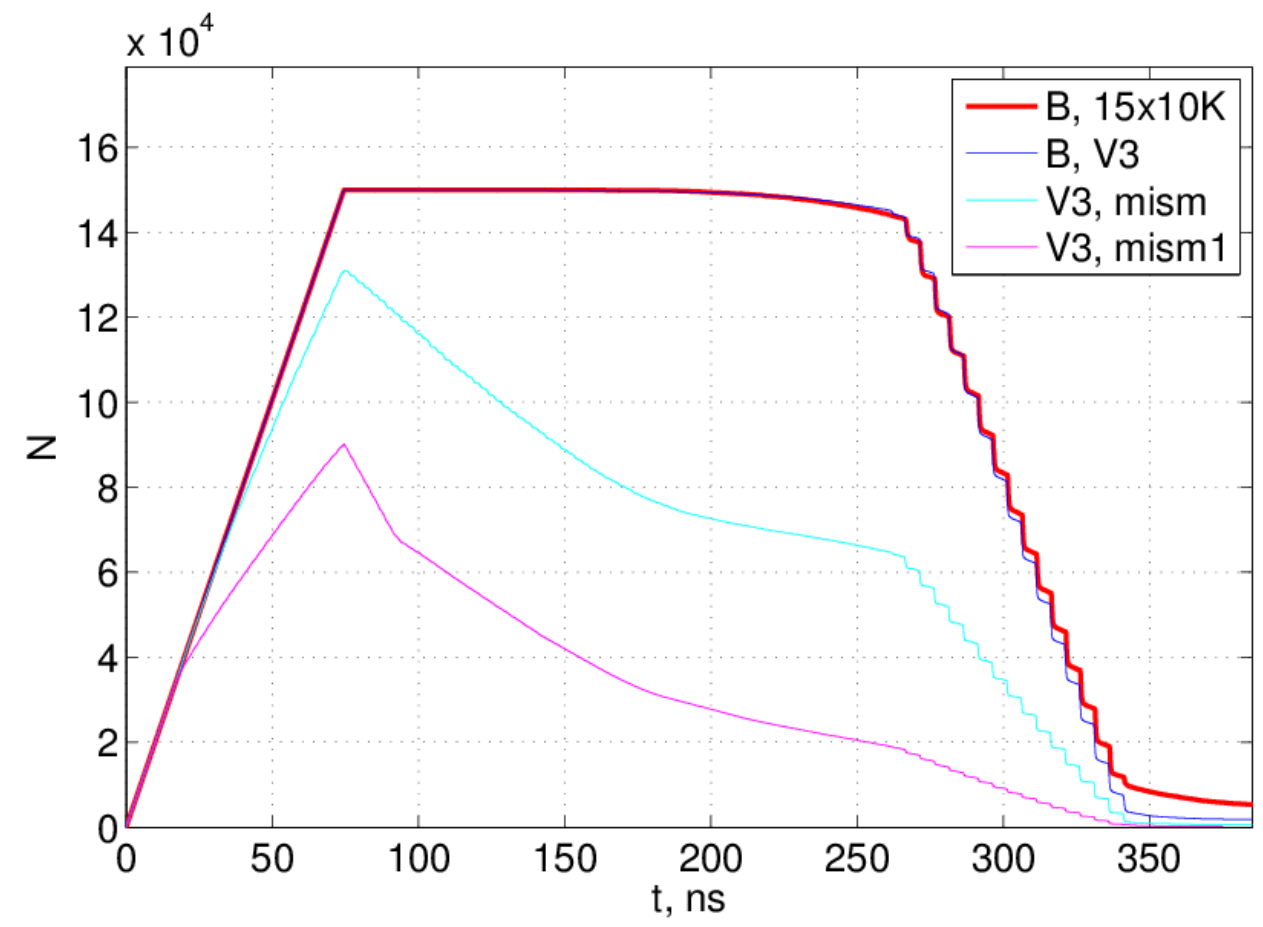

Figure 21: Number of particles in the RFQ model B versus time from a few different runs with 60-mA $\mathrm{CW} \mathrm{H} \mathrm{H}^{-} 15 \times 10 \mathrm{~K}$ beam.

For these cases, the initial transverse normalized rms emittances were $0.11 \pi \mathrm{mm} \cdot \mathrm{mrad}$ in the vertical plane and $0.15 \pi \mathrm{mm} \cdot \mathrm{mrad}$ in the horizontal one $(0.3 \pi \mathrm{mm} \cdot \mathrm{mrad}$ in the matched case $)$, but the beam parameters that we inferred from the measurements were different from the design values. In the last case in Fig. 21, marked "V3, mism1", the initial distribution was additionally rotated $45^{\circ}$ in the transverse plane plus the initial energy was $37 \mathrm{keV}$ instead of $35 \mathrm{keV}$. The transmission in the case "V3, mism" was $37 \%$ for 5 central bunches, and much lower in the last case. This comparison suggests that matching the initial distribution to the RFQ is very important, while the voltage profile has some influence but not critical for transmission. It is worth mentioning that the FNAL RFQ transmission achieved in operation so far has been around $60-70 \%[8]$.

We discussed above the effects of the RF fields in the RFQ exit gap on the beam. Similar RF fields exist in the entrance gap, see [1], and they influence the CW beam being injected in the RFQ. Estimates in [1] suggest that the resulting beam-energy change after the entrance gap should be small due to a very small transit-time factor. Here we look at this problem using PIC simulation results. Figure 22 shows the longitudinal phase space $x$ - $W$ near the RFQ entrance in model B: five snapshots taken with 1-ns step cover time interval of one RF period. The left picture corresponds to snapshots starting from $t=301 \mathrm{~ns}$ in the model B with nominal inter-vane voltage and 70x10K beam. In fact, this is just a fragment of the full phase-space picture similar to Fig. 4 but for model B - its bottom-left corner - while the other fragment (top-right) is shown in Fig. 5 (bottom); the matching colors correspond to the same times. One can see that the CW beam is injected at $x=-630 \mathrm{~mm}$ with the energy of $35 \mathrm{keV}$. The energy spread of about $1 \mathrm{keV}$ is due to space charge. As particles cross the entrance gap - interval $-600 \mathrm{~mm}<\mathrm{x}<-593$ $\mathrm{mm}$, if one counts only to the beginning of the radial matching section of the vanes - the particle energy changes, either increasing or decreasing depending on the RF field phase, with an amplitude around 4-5 $\mathrm{keV}$. However, a few $\mathrm{cm}$ after the gap this energy modulation becomes much smaller, comparable to the space-charge energy spread. 

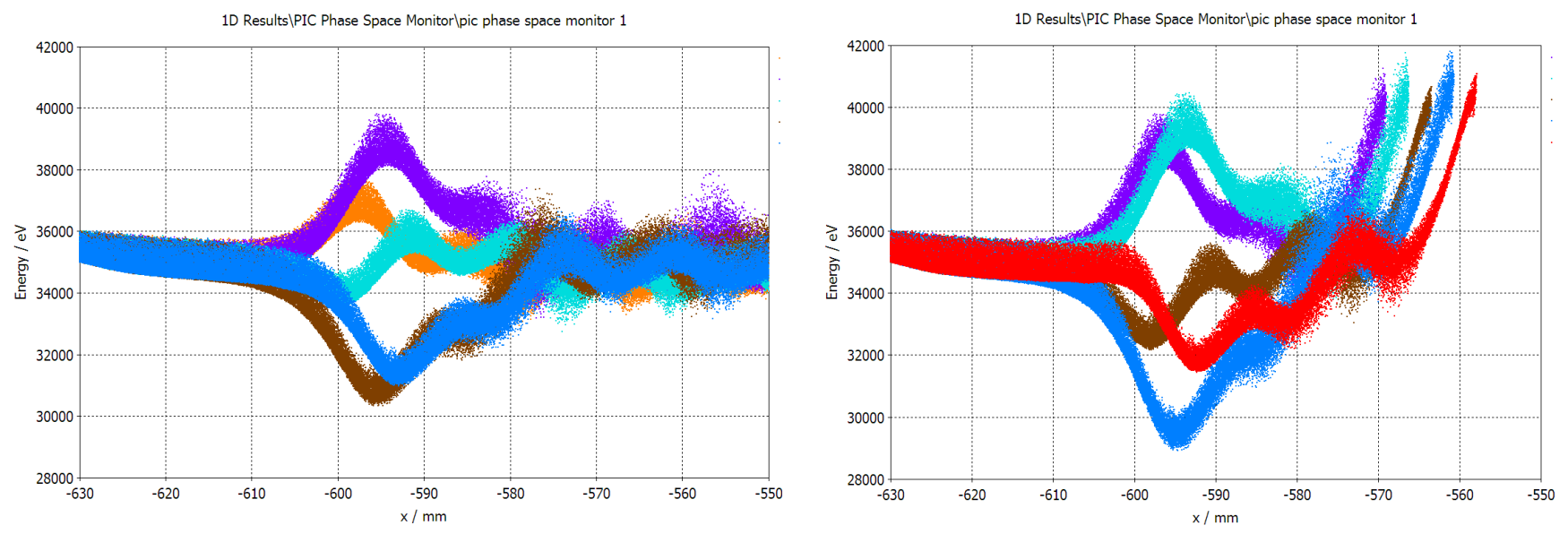

Figure 22: Five overlapping snapshots of the phase space with 1-ns step near the RFQ entrance in model B with $70 \mathrm{x} 10 \mathrm{~K}$ beam (cf. Fig. 5, same times $=$ colors, SW corner) - left; same with $V=1.25 V_{0}-$ right.

The right picture in Fig. 22 shows similar phase-space snapshots but in the model B with inter-vane voltage $V=1.25 V_{0}$. The amplitude of the energy modulation in the gap increases to $5-6 \mathrm{keV}$, as one can expect. The snapshots in Fig. 22 (right) correspond to simulation times starting at $t=23 \mathrm{~ns}$, so the beam leading particles are visible as they propagate along the RFQ structure. Notice that they have somewhat higher energy because of a push from the longitudinal space charge. The matched beam injection in model $\mathrm{B}$ is illustrated by 3 -D particle-monitor results at a later moment, $t \approx 75 \mathrm{~ns}$, in Fig. 23.



Figure 23: Particles near the entrance in the RFQ model B at $t \approx 75 \mathrm{~ns}$ with $15 \mathrm{RF}$ periods of $70 \times 10 \mathrm{~K}$ beam injected. Energy modulation in the entrance gap practically disappears before bunching starts. 
One can see that the injected $\mathrm{CW}$ beam converges to provide a waist near the vane entrance. Notice also the beam energy modulation indicated by color: at the shown moment, the beam in the gap has the energy lower than at injecting. This gap-induced energy modulation disappears further in the structure and even further, when the beam enters the buncher section, another energy modulation is produced by the RF fields there. Using 2-D particle monitors, we have traced how the energy (velocity) modulation in the gap translates into a modulation of the beam longitudinal density further down the structure. In the shown case, however, these modulation effects are somewhat smeared by space charge. Still, one can imagine designing an entrance gap in such a way that is produces such a pre-bunching of the injected CW beam that will facilitate further bunch formation in the RFQ.

In conclusion of this section, we describe some computational details. Following a well-developed procedure [10], we import MWS-computed and properly scaled 3-D RF fields, see in [1], into the Particle Studio (PS) PIC solver. The mesh used in the PS can be different from the one employed in the MWS to calculate fields with eigensolvers, but both were about the same size. Actually, even the model dimensions in MWS and PS can be different, assuming that the regions were RF fields are important overlap, which allowed us to adjust the beam pipes in PS models. The PIC solver does not allow partially filled (TST) mesh cells, so some mesh cells and faces, usually the ones near complicated metal boundaries, end up being filled by perfect electric conductor (PEC) in the meshing process. However, this does not influence the quality of imported MWS fields that were computed with the AKS eigensolver that allows TST cells. As long as such PEC cells or faces are not in the beam path, the PIC results can be trusted. Surprisingly, the PIC simulation times for this problem turned out to be practically independent of the number of particles used in a run, up to $700 \mathrm{~K}$. One possible explanation is that the overhead due to handling large imported files containing the external 3-D RF MWS fields, over $100 \mathrm{MB}$, exceeds that of many particles. A long PS PIC run on a mesh with 5.25 million mesh points and $70 \times 10 \mathrm{~K}$ input beam takes $\sim 40$ hours on a $3+$-year-old $\mathrm{PC}$ with dual quad-core $3.33-\mathrm{GHz}$ Xeon W5590 processors and 24 GB of memory. It also produces more than $20 \mathrm{~GB}$ of data on disk. The CST PIC solver is well parallelized; the computer memory was not a limitation is this case. The PIC run time depends directly on the chosen length of simulation time, $700 \mathrm{~ns}$ in this case, but not strongly on the particle number. On the other hand, the amount of disk space to store the run results depends directly on the number of particles, mainly due to huge 3-D particle monitor files: with snapshots every $1 \mathrm{~ns}$, the total is $18 \mathrm{~GB}$ in the above case. However, an animated sequence of such snapshots makes a nice movie that well illustrates how an RFQ works.

\section{Summary.}

Particle-in-cell (PIC) modeling of beam dynamics in the 4-rod FNAL RFQ has been performed with the CST Particle Studio (PS). Starting from a detailed engineering CAD model of the RFQ, its RF fields were calculated [1] using the CST MicroWave Studio (MWS). These RF fields were then imported in the PS model of the RFQ, and PIC modeling of beam dynamics with various initial beam distributions was carried out.

Some simulation results are presented in Sec. 3. Of particular interest are effects associated with 3-D field asymmetries and end-gap fields, which are not predicted by standard RFQ design codes. We found that depending on the diameter of the exit beam pipe, the RFQ output energy can change due to the endgap fields. In the case of a narrow beam pipe (model B), which approximately corresponds to the layout of the FNAL RFQ box with its end plates installed, the energy was found to drop by about $20 \mathrm{keV}$ below the design value of $750 \mathrm{keV}$ produced by the vane modulations. In the RFQ model A, with wide beam pipes, there was no energy drop. The end plates are installed on the inner end walls of the FNAL 
RFQ resonator to prevent RF leakage into beam pipes. The end plate closes a wide opening (diameter $100 \mathrm{~mm}$ ) of the RFQ-box flange and has a small (diameter $20 \mathrm{~mm}$ ) beam hole. The model A in our simulations approximates the RFQ resonator without end plates. Actually, this modeling project was stimulated by discussions with our FNAL colleagues at IPAC'12 conference (May 2012) where we learned that they observe the low RFQ output energy, $\sim 710 \mathrm{keV}$. The results of initial PS simulation runs in June 2012 led us to suggest that changing the geometry of the RFQ box by increasing the exitbeam-pipe size could help. Fortunately, such a change was possible to implement relatively easy by removing the end plate near the exit of the RFQ box. When this change was implemented by our FNAL colleagues by the end of June 2012, the measured RFQ output energy increased to $756 \mathrm{keV}[9,6]$.

In a similar way, our analysis of the exit beam angle distributions followed the FNAL observation [8] of beam loss in the MEBT after the RFQ, which our FNAL colleagues traced to the beam exiting RFQ at an angle of $1-2^{\circ}$ vertically from the axis, in December 2012. We analyzed the data from our previous PS runs and confirmed such an effect in simulations, see Sec. 3. Finally, our PS results suggest that the beam matching to the RFQ entrance has the strongest influence on the RFQ beam transmission.

Overall, the experience acquired in the process of FNAL RFQ simulation with the CST Studio codes better prepares us to evaluate performance of a new 4-rod RFQ for the LANSCE accelerator complex.

\section{Acknowledgements}

The author would like to thank Larry Rybarcyk, Bob Garnett, Yuri Batygin, and Tom Wangler of LANL; Cheng-Yang Tan, William Pellico, and Gennady Romanov of FNAL; Janet Schmidt and Alwin Schempp of Goethe University (Frankfurt, Germany) for their kind help, sage advices, useful information, and stimulating discussions.

\section{References.}

1. S. Kurennoy. Tech note AOT-ABS: 2012- 017 (TN); LA-UR-12-26388, Los Alamos, 2012.

2. CST Studio Suite, CST, 2012, www.cst.com

3. R.W. Garnett, et al. IPAC11 (2011), p. 2658.

4. C.-Y. Tan et al. PAC11 (2011), p. 1701.

5. Los Alamos Accelerator Code Group, laacg.lanl.gov

6. "FNAL RFQ Injector Upgrade Project" (update v11b, Jan. 2013), http://beamdocs.fnal.gov/ADpublic/DocDB/ShowDocument?docid=3646

7. L.J. Rybarcyk, Private communication, 2012.

8. C.-Y. Tan, Private communication, Dec. 2012.

9. C.-Y. Tan, Private communications, May-Oct. 2012.

10. S.S. Kurennoy, L.J. Rybarcyk, J.F. O'Hara, E.R. Olivas, T. P. Wangler. "H-mode accelerating structures with PMQ beam focusing." Phys. Rev. ST Accel. Beams, 15, 090101 (2012). 